\title{
Bilateral Bulging on Pharyngeal Contraction
}

National Cancer Institute

\section{Source}

National Cancer Institute. Bilateral Bulging on Pharyngeal Contraction. NCI Thesaurus.

Code C127318.

A finding of bilateral bulging on pharyngeal contraction during swallowing. 